\title{
El Texto de La Araucana de Alonso de Ercilla: Observaciones a la Edición de José Toribio Medina
}

Es materia fuera de discusión el valor erudito de la edición del poema de don Alonso de Ercilla que el polígrafo chileno José Toribio Medina publicó entre 1910 y 1918 en Santiago de Chile. No ha pasado el tiempo para sus veintitrés Ilustraciones ni ha disminuido el interés de los datos e información histórico-crítica acumulados en los cinco grandes tomos que componen la edición, bien calificada de "monumental"'. 1 .

Conviene, sin embargo, puntualizar algunas de las características del texto del poema que presenta la edición, como contribución a su mejor conocimiento y como una forma de homenaje a la labor extraordinaria de Medina. Como todas las ediciones modernas se han apoyado hasta ahora en este texto, no será inútil señalar los riesgos que esta confianza supone.

En los ocho años en que se fueron publicando los tomos, Medina varió criterios y planes iniciales. Ciertos cambios carecen de importancia final, pero reflejan indecisiones sobre la marcha; por ejemplo, el orden de las Ilustraciones pasó por diversas modificaciones de acuerdo con la rapidez con que unas se adelantaban o otras planeadas anteriormente.

Así, se anuncia en la contratapa exterior del Tomo II (1913) un orden que no fue mantenido y algunas Ilustraciones fueron refundidas con otro título. Las planeadas Ilustraciones VI y VII, "Ercilla juzgado por La Araucana", y "Ercilla en el teatro" respectivamente, pasaron a ser Ilustración XVIII, "Ercilla y sus héroes en la literatura". En la nota "Al lector" que inicia el Tomo II, advertía Medina que-la gran cantidad de nuevos documentos aparecidos después de la edición de la RAE (1866)'le había obligado a ponerlos en tomo aparte (p.V); esta abundancia de nueva información explica que la planeada Ilustración XIX, "Indice alfabético de personas", quedara desglosada en dos Indices que pasaron a ser luego las Ilustraciones VIII y IX respectivamente; en realidad estos dos Indices no llevan números, como tampoco el previo "Indice geográfico de La Araucana', pero debe deducirse este orden pues el cuarto tomo (segundo de las Ilustraciones) se inicia en la Ilustración $\mathrm{X}$.

1 F. Pierce. La poesta épica española del Siglo de Oro. Madrid, 1968. Segunda edición revisada y aumentada, p. 187. 
En cuanto al texto mismo del poema, los problemas que presenta son más complejos porque las declaraciones de Medina son contradictorias o ambiguas. En la "Advertencia del editor", sin duda escrita mucho antes de su impresión en el Tomo I, "Texto", publicado en 1910, aclara Medina (p.VIII):

Para la publicación del texto hemos seguido el de la edición de Madrid de 15891590 , última que salió en aquella ciudad en vida de Ercilla y la cual sin duda fue impresa bajo la inmediata inspección del poeta, (...) En cuanto a los cantos agregados por primera vez en la edición madrileña de 1597 del Licenciado Castro, no podiamos en esta parte, elegir otra, como que, según opinión corriente, para ella debió tener a la vista los borradores del autor, los cuales fueron, a lo que se cree, entregados por la viuda al editor. ${ }^{2}$

Hay que entender el texto citado de Medina en un sentido bastante amplio, pues "seguir la edición de Madrid de 1589-1590" no significa transcribirlo rigurosamente. En efectọ, Medina modernizó la puntuación y la ortografia, y en la Ilustración XIII, "Variantes de La Araucana', señala la supresión de la ss y la modernización del uso ortográfico de la $x$ (Tomo IV. p. 108), pero advierte que "se conservan, en cambio, las voces anticuadas en su forma más o menos radical, como aun en su escritura" (ibid.). Y más adelante insiste: "debimos optar por seguir el texto tal como le hallamos sin atrevernos a tocar palabra, respetando hasta lo último la integridad del original'.

Sin embargo, el examen del texto de $\mathrm{F}$ permite advertir numerosas discrepancias, lo que obliga a suponer que Medina manejó con bastante libertad y criterio personal la fidelidad al texto, o que el "original" bien puede ser otro. En efecto, Medina transcribió el texto de otra edición del poema de Ercilla. En la Illustración X, "Bibliografía de $\mathrm{La}$ A raucana", aparecida en el Tomo IV, 1917, al describir la edición de la RAE (Madrid, 1866), comenta Medina que "esta académica había sido la que nosotros adoptamos para el nuestro casi literalmente",3 (p. 52), y añade, más adelante, "reservándonos, eso sí, admitir las modificaciones y enmiendas que parecía de todo punto indispensable ejecutar". Pero qué texto es el que sigue la edición de la RAE y cuáles fueron las modificaciones y enmiendas deMedina es la pregunta inevitable. El texto es $\mathrm{H}$, y para justificar la elección, Medina se basa en las palabras que el editor de H, Ferrer del Río, dedica a la edición en la Ilustración IX del segundo tomo de su propia edición: "Por superior a todas como correcta se debe considerar sin duda la hecha en Madrid el año 1597 a costa de Miguel

\footnotetext{
${ }^{2}$ Para simplificar las denominaciones seguimos el siguiente sistema de abreviaturas:

A Primera Parte, Madrid, 1569. B: Primera y Segunda Partes, Madrid, 1578 (en octavo). C: Primera y Segunda Partes, Madrid, 1578 (en cuarto). D: Segunda Parte, Zaragoza, 1578. E: Tercera Parte, Madrid, 1589. F: Primera, Segunda y Tercera Partes, Madrid, 1589-1590. G: Primera, Segunda y Tercera Partes, Barcelona, 1592. H: Primera, Segunda y Tercera Partes, Madrid, 1597. I: Primera, Segunda y Tercera Partes, Amberes, 1597.

Los números de las citas corresponden, respectivamente, a Canto, octava y verso. Hemos abreviado Keniston: Hayward Keniston, The Syntax of Castilian Prose, The Sixteenth Century, Chicago, 1937.

${ }^{3}$ V. comentario de S. Dinamarca "Los estudios de Medina sobre Ercilla", Atenea, XXIX,t.CVII, n. 327-328 (set. -oct. 1952) 341-379, p. 349.
} 
Martínez, y en casa del Licenciado Castro, donde se advierten oportunas enmiendas de Ercilla, según todas las verosimilitudes"' (p. 459) ${ }^{4}$. Pero Ferrer del Río también introdujo modificaciones al texto de $\mathrm{H}$, que en muchos casos aceptó Medina.

Hemos notado alrededor de doscientos cincuenta cambios en el texto de $\mathrm{H}$ introducidos por Ferrer del Río que pasaron a la edición de Medina; algunos son correcciones de erratas evidentes, otros son modernizaciones ortográficas o fonéticas y el resto claras modificaciones.

Las erratas evidentes, ya corregidas por Ferrer del Río, en muchos casos no fueron transcriptas en las variantes por Medina. Hay por lo menos cincuenta lecturas que deben considerarse erratas o tal vez innovaciones de $\mathrm{H}^{5}$, y de ellas, más de la mitad quedan sin registrar en Medina, sin duda porque ya Ferrer del Río las había corregido. En los casos restantes no siempre aparece $\mathrm{H}$ señalada en las variantes y puede sospecharse que $\mathrm{H}$ fue leída con menos cuidado dadas las seguridades que ofrecía el texto académico ${ }^{6}$.

Las variantes fonéticas seguidas por Medina son, en general, modernizaciones: enebro por nebro $(3,65,8)$, adarga por darga $(5,10,5 ; 6,54,4)$, polvareda por polvoreda $(5,29,5)$, fe por fee $(32,55,5)$, ven por veen $(3,48,6)$, Perí por Pirú $(12,89,1)$, que alcanzan a apellidos y otras regiones geográficas: Croacia $(18,45,8), \operatorname{Croatos}(24,4,1)$, Transilvanos (24, 4, 2), Georgianos (24, 4, 4), Sarracina (24, 84, 2), Villagrán (15, 36, 1), Biezma (25, 58, 2), Ibarra $(25,58,3)$, Ruiz $(25,58,8)$, Gredosia $(27,9,5)$, Alejandro(27,15, 6), Cremona (27, 25, 5), Trujillo(27, 45, 5), Augusto Octaviano (32, $46,3)$.

Medina sigue a Ferrer del Río en la unificación de vacilaciones vocálicas comunes en la conjunción $(11,56,8 ; 23,46,4 ; 28,51,4)$, en formas verbales: diferirlo $(2,8,1)$, crujiendo $(10,21,5)$, decida $(11,21,7)$, dividiera $(25,52,4)$, o en formas nominales: turbulento $(4,63,7)$, disgusto $(4,98,5)$, intrinseco $(9,60,1 ; 30,60,2 ; 37,48,5)$, impetu $(15,74,4)$, investidura $(17,53,7)$, rencores $(20,5,3)$, coligados $(23,85,8)$, estrépito $(26,13,3)$, asimismo $(29,15,3)$, codicia $(32,55,7 ; 32,56,6)$.

También sigue Medina a la edición académica en la transcripción de contracciones; en la Ilustración XIII (Tomo IV, p.108) señala la separación en dos palabras de quel, sobrello y entrellos; la lista debe extenderse a dellos, desta, deste, desto, ques y aun se puede agregar lagua $(22,32,8)$. También, como hace Ferrer del Río, despliega la a del objeto animado en casos como a aquel, a alguno, con lo que se añade al problema ortográfico un cambio de los usos sintácticos de la época que implica también modernización.

La modernización ortográfica en el uso de la letra $x$ (Tomo IV, p. 108) termina por confundirse con la modernización fonética en el caso de las prepalatales y así se transcribe ejecución $(30,4,5 ; 31,16,8)$ y desencasarse ultracorrige en desencajar $(16,32,8 ; 19$, $45,8)$. Si en el caso de dieciseis $(16,39,2)$ la variante es poco importante, el cambio

\footnotetext{
${ }^{4}$ En The New York Public Library hay un ejemplar con variante de portada; Miguel Martínez aparece reemplazado por Juan de Montoya.

5 Pueden considerarse innovaciones textuales de $\mathrm{H}$ sin tradición previa: 2, 82, 4: y araucano; 4, 15, 2 : cercaron; $5,51,6: y$ ruido $; 24,56,1$ : resolviendo $; 26,47,1:$ bermoso, verde $; 27,40,3:$ y lucida costa y versos enteros como 3, 63, 1-6.

${ }^{6}$ De todos modos, algunas erratas de $\mathrm{H}$ no advertidas por Ferrer del Río fueron prolijamente enmendadas por Medina; así, por ejemplo, 1,34,5 (en dilatarse).
} 
resulta arbitrario en bijadeando $(4,44,3)$, que se transcribe $y$ jadeando. La afirmación de que "debimos optar por seguir el texto tal como lo hallamos, sin atrevernos a tocar palabra, respetando hasta lo último la integridad del original" (Tomo IV, p. 108) debe entenderse en el sentido muy amplio de reemplazo de una palabra por otra y en su estructura fonética. En efecto, con Ferrer del Río, Medina moderniza también los grupos consonánticos cultos $c c, c t, m n, n n$ y aparecen unidos a penas, de espacio, a caso, a bajo. En dos casos hemos notado que los cambios en la puntuación modifican el sentido del texto $(32,79,2 ; 33,31$, 7).

Los cambios textuales propiamente dichos que la edición académica introdujo en el texto de $\mathrm{H}$ y que Medina aceptó son de diverso tipo; van desde correcciones de supuestas faltas de concordancia sintáctica, bastante frecuentes en la literatura de la época, hasta cambios sintácticos y de sentido generalmente innecesarios. En muchos casos, como se verá, el cambio supone la vuelta a una edición previa y el resultado es una fusión textual improcedente y una técnica muy libre en el manejo de las variantes.

Entre los cambios debidos a supuestas faltas de concordancia hemos notado ban de ir $(1,15,3)$ contra ba de ir de B,C,F, G,H,I, que se explica porque se refiere a cada niño de los que se habla en el verso 1 de la octava; pérdidas $(1,59,1)$ se aparta de $\mathrm{H}$, pérdida, para seguir la lectura de F; $l o(4,32,6)$ va contra los de A, B, C, F, G, H, I, que se explica porque se refiere a la proposición objeto del verso anterior, con valor colectivo 'las cosas que...'; usados $(2,12,6)$ sigue el texto de A, B, C, G, I, pero $\mathrm{F}$ y $\mathrm{H}$ traen usado porque concuerda con Cayocupil del primer verso; se puede $(7,50,7)$ de A, B, C, F, H, I se explica por el sentido colectivo de la proposición e infinitivos anteriores y no hace falta la forma plural propuesta por Ferrer del Río; ganoso $(8,9,7)$ vuelve al texto de A y de B, pero el ganosos de $\mathrm{H}$ y otras ediciones consultadas se explica perfectamente por el sentido colectivo de campo 'campamento'; celebran $(8,11,8)$ concuerda en plural con el colectivo gente, y el cambio a singular es innecesario; merece $(8,54,4)$ de todas las ediciones antiguas concuerda en singular con dos sustantivos abstractos casi sinónimos, como es frecuente en la literatura de la época (Keniston, 36.41), y la forma plural es innecesaria; la ordena $(18,57,2)$ concuerda con punición del verso 6 de la estrofa y la forma propuesta resulta innecesaria; contarlos $(19,50,8)$ concuerda en B, C, D, F, G, H, I con daño de la octava siguiente y la forma pronominal femenina no es necesaria; le $(22,26,8)$ de $\mathrm{B}, \mathrm{C}$, D, F,G,H,I concuerda en sentido con "gente española"'sobreentendido en paralelismo con el "araucana gente" del primer verso y no hace falta la forma plural propuesta.

Los cambios en el aspecto sintáctico llevados a cabo por Ferrer del Río y aceptados por Medina tienden a eliminar características de la lengua literaria del siglo XVI y no parecen justificados.'Do a las $(1,59,5)$ de A,B,C,F, G,H,I se explica por el régimen de traer del verso siguiente y la supresión de $a$ deja sin sentido el texto; le asegura $(2,33,6)$ de todas las ediciones antiguas consultadas es caso de leísmo que no necesita ser corregido (Keniston, 7.132); otros casos de cambios innecesarios de usos pronominales en 4, 7, 3 (le leyere), 22, 12, 4 (Haciéndolos),'(25, 28, 6)(Lo sale) y 25, 46, 6 (Lo rodea); que en una $(2,37,3)$ agrega un innecesario en pues es obvio que bubiese del sexto verso significa 'tuviese'; a toda priesa $(2,52,2)$ cambia el original todo en concordancia con el sujeto claro dia (Keniston, 39.96); acertar a $(3,6,7)$ es uso normaly no se explica la supresión de la preposición en que a la derecha via de todas las ediciones consultadas; el cambio de 
juntos al general $(4,78,1)$ de todas las ediciones consultadas por juntos, el general no se justifica pues juntos en este texto es el participio del verbo juntar; el cambio de sin orden $y$ $(6,14,3)$ de todas las ediciones a sin orden ni no respeta usos sintácticos de la época (Keniston, 40.94); la entregasen $(9,11,5)$ de A, B, C, F, G, H, I concuerda en género con la Imperial, mencionada en las octavas 6 y 7 del Canto y el cambio a le es innecesario; el adjetivo alerto $(9,81,6)$ es usual en la época (V. ej. en Autoridades) y nada explica el cambio contra el texto de todas las ediciones antiguas; infante $(18,52,3)$ como forma femenina latinizante (infanta aparece muy temprano en español; cf. Salvador Fernández, Gramática española, p. 158) aparece ya en $\mathrm{C}$ antes de $\mathrm{H}$ y no necesita cambiarse a infanta. La ciñera la ufana y leda frente $(20,47,7)$ se explica como caso de pronombre redundante (Keniston, 8.822) y no es necesario el reemplazo por le (ya en B y D); frenos es la lectura de todas las ediciones consultadas, y el singular para mantener el paralelismo formal con el calcaño anterior, no justifica una enmienda; no hace falta quitar a al verso: Luego, a la voz de Rengo obedecida $(22,43,1)$ pues el participio significa acá 'que se obedece' y no se trata de una construcción de participio absoluto; la omisión de $a$ ante sustantivo propio que funciona como objeto directo, sobre todo si comienza por vocal, no es desconocida, de modo que la preposición agregada parece innecesaria ; en 25, 56, 6 el objeto directo con preposición (para cosa) se justifica por los dos pronombres indefinidos cercanos y ya está en $\mathrm{D}$; tanto el arte $(28,48,1)$ es la lectura correcta y la forma femenina para el adjetivo no aparece en las ediciones consultadas; las ediciones antiguas consultadas traen en una (32, 26,6 ), que debe entenderse en concordancia con casa de la octava siguiente y el cambio a la forma masculina es innecesario.

Los cambios de sentido en algunos casos se explican por errónea interpretación del texto de $\mathrm{H}$; en otros, por preferencia de una edición previa. En 1, 9, 1 piloto se opone arbitrariamente a la tradición textual que trae siempre pilotos; medir la pica $(1,23,8)$ de A, B, C,F, G, H,I es frase hecha y no se explica el cambio del artículo por la preposición a que se lee en la edición académica y acepta Medina; $Y$ así a cualquier $(1,33,5)$ de B,F,G,H,I se explica como ' $Y$ así sucede a cualquier señor'y la supresión de la preposición parece superflua; armas $(2,20,4)$ de $\mathrm{H}$ por ramas de las ediciones anteriores e I, parece atractiva corrección que aclara este texto obscuro, si se entiende el desgajando anterior 'arrancando violentamente', pero Ferrer del Río, y así Medina, prefirió apartarse del texto para evitar una repetición molesta de armas, aunque con desventaja para el sentido del verso; dañoso $(3,66,1)$ deltodas las ediciones es adjetivo muy usado en la lírica de la época y el cambio a furioso es inexplicable; el agregado de más de $(4,30,4)$, sin duda por razones métricas, es arbitrario: el verso tiene aspiración en bizo, y viudas es trisilaba; en $4,77,2$, la vuelta al texto de $B$ no parece necesaria si se tiene en cuenta el hiato en $s u$ ejército; el cambio de osado $(5,34,7)$ de todas las ediciones por usado es arbitrario y tal vez se explique por rechazo de la repetición (falsa, como se verá) de usado, ya en el verso anterior; nada justifica el cambio de planto $(6,19,3)$ de todas las ediciones a llanto: Autoridades trae ejemplo de este latinismo en el contemporáneo fray Luis de Granada; nada justifica el paso de La fuerza $(6,32,4)$ presente en todas las ediciones al plural; estos $(6,51,1)$ se refiere al señores de la octava anterior y el cambio a esto, que totaliza la situación descripta antes, no se justifica; Tal vez bay que $(7,16,3)$ debe entenderse 'a veces sucede que' (V. DCELC s.v. tal) y no hace falta cambiar a voz; nada impide mantener 
la lectura $Y o$ dije $(9,6,1)$ de todas las ediciones, que se cambia a un arbitrario $Y a$ dije; la vuelta al texto de A,B,C en 10,10,6 no justifica el cambio de $\mathrm{H}$ presa entre la gente a presa alegremente; asistiese $(10,11,4)$ de $\mathrm{F}$ y $\mathrm{H}$ no necesita cambiarse por estuviese del resto de las ediciones; la vuelta al texto de A Tras esto $(12,60,5)$ por Tras estos del resto de las ediciones no se justifica, pues el plural concuerda con briosos y aquellos; nuestra $(16,65,2)$ de todas las ediciones concuerda con desbonra y no con banderas, de modo que la forma plural no parece necesaria; en 18, 13, 5 Ercilla usa prisión en la acepción 'prisionero' como otras veces en el poema $(26,21 ; 7$ por ejemplo), de modo que el agregado de en deja sin sentido el texto de todas las ediciones; el cambio de fugitivas velas $(18,44,7)$ de $\mathrm{H}$ a fatigadas velas se explicaría porque es lectura que aparece en el resto de las ediciones, pero funde dos textos innecesariamente; falsa y fementida $(18,55,8)$ es un caso de repetición sinonímica con valor intensificador muy frecuente en Ercilla y la lectura fe mentida, tal vez sugerida por $\mathrm{C}$, no parece necesaria; Por miedo $(19,38,8)$ parece mejor lectura que Por medio de las armas y enemigos, ya en F, G,I, que no tiene sentido; espalda $(19,43,6)$, ya en $\mathrm{B}, \mathrm{C}, \mathrm{D}$, altera sin necesidad el texto de $\mathrm{H}$, en donde se lee espada; en 21, 7, 2 incrédulo 'increíble' es latinismo de sentido y no es preciso acudir a una concordancia femenina con el sujeto Ella ya propuesta por cuatro ediciones antiguas: la palabra era de uso literario relativamente nuevo (cf. DCELC s.v. creer, con autoridades para crédulo solamente); en $\mathrm{G}, \mathrm{H}, \mathrm{I}$ aparece bruto sangriento $(22,44,3)$ y no se explica su reemplazo por todo sangriento, que es la lectura primitiva de B,C,D; A cada priesa (24, $61,6)$ no necesita corrección pues priesa es aquí 'aprieto'; la variante $A c a$ da está tomada de C, D; nada justifica el cambio de Mira en Africa, el Sur $(27,16,1)$ presente en otras ediciones antiguas por al Sur ya en B y D.

En la Ilustración X 'Bibliografía de La Araucana' (tomo IV, p. 52) advertía Medina que se reservaba el derecho de "admitir modificaciones y enmiendas que parecía de todo punto indispensable ejecutar' a la edición académica y el examen de los dos textos nos ha permitido observar buen número de discrepancias que hacen posible asegurar que el método de transcripción adoptado por Medina tendía al eclecticismo. Medina ha modernizado los nombres propios, a veces excesivamente, pues las formas de RAE son las que se leen en las ediciones del siglo XVI; notamos Pablo frente a Paulo (7, 30,1), Marcos Veas por Marco Veaz (12, Título), pilmaiquenes por plimaiquenes $(21,49,1)$, Yanaruna ya en $F$ por Ynarauna (25, 31, 2), Tamorlán por Taborlán $(27,13,6)$, Vulturno por Volturno (27, 43, 8), Codro por Cosdro (29, 2, 7).

En cambio, fonéticamente, Medina se aparta de la edición académica y también "envejece", el texto. Esto se nota en el tratamiento de las vocales átonas en que la forma elegida es, a veces, la que ha desaparecido: cudicia $(2,92,7$ y 2, 93, 8) por codicia que se lee ya en $\mathrm{H}$; preveniendo $(18,35,2)$ por previniendo de la edición transcripta; devisas $(23,78,1)$ por divisas. Casos semejantes se hallan en grupos consonánticos nominales, pronominales y verbales: eleción $(2,62,2)$ por elección; comigo $(12,24,5)$ por conmigo; reduzga $(20,53,2)$ por reduzca; jatanciosos $(29,19,8$ con errata jataciosos) por jactanciosos.

También hay modernizaciones que escaparon al escrupuloso examen de Ferrer del Rio: recibido $(2,59,3)$ por recebido; estropeados $(22,17,7)$ por estropiados; asimismo $(25,47,1)$, por asimesmo; oblicua $(28,54,3)$ por oblica; transportada $(8,45,6)$ por 
trasportada; transparente $(23,66,2$ y $23,76,2)$ por trasparente. Estas modernizaciones alcanzan a fenómenos de fonética sintáctica como corre a abrazar $(8,10,8)$, poricorre abrazar; vengo a asegurar $(17,8,5)$ por vengo asegurar; a abordarse $(24,48,2)$ por a bordarse; pero el fenómeno opuesto no está ausente: Quel $(12,42,7)$ por Que el.

Estos aspectos de la transcripción textual dan indicio del vacilante sistema elegido y permiten suponer modificaciones aún más sustanciales. En efecto Medina exageró la tarea de variación arduamente iniciada por Ferrer del Río y añadió buen número de cambios gramaticales y de sentido a los ya perpetrados. Por lo menos nueve deben de ser erratas de impresión; en 21,39,7 y poblado por y muy poblado con pérdida de una sílaba del verso; en 31,26,2 Medina transcribe en la mitad pero el agregado del artículo añade una silaba al endecasílabo y debe considerarse errata; lo mismo puede decirse de dentro de $(3,80,6)$ por dentro en que destruye la métrica por sílaba de más y lo opuesto, por sílaba de menos, en 18 , 39,1 al suprimir en en Luego en el; considerando $(33,19,5)$ debe ser errata por considerado, como en todas las ediciones consultadas, pues quiebra la rima con intimado del segundo verso de la octava; Samogocia $(27,29,2)$ por Samagocia de H y, más moderno, Samogicia en Ferrer del Río; en 26,33,8 falta la palabra asi, indispensable para la métrica; emboscando $(27,20,4)$ por embocando, de todas las ediciones consultadas, no tiene sentido y también debe considerarse errata.

Entre los cambios sintácticos, el más frecuente corresponde a cambios de número; hemos notado once pluralizaciones y cinco vueltas a la forma singular, contra la tradición textual y la edición de Ferrer del Río. En los cambios al plural, tres aparecen en formas verbales: iban $(9,88,7)$ por $i b a$ de las ediciones consultadas, concuerda con quien distributivo del primer verso de la octava; conocen $(11,25,3)$ por conoces de todas las ediciones consultadas; eran $(21,27,6)$ por era, que se explica porque concuerda con el colectivo gente del segundo verso de la octava. Seis casos no justifican el cambio ni en la tradición textual ni en una mejor comprensión del texto: otros servicios $(1,18,2)$, limites $(3,2,6)$, las tierras $(3,21,7)$, acidentes $(7,7,6)$, tantas glorias $(23,9,2)$, a términos $(32,50,5)$. En un séptimo texto, el cambio es directamente un error: los sintieron $(11,71,7)$ deja sin referente lógico al pronombre que en la forma singular repite el rico cuerno del verso anterior. En el octavo caso $(26,4,8)$, el cambio a plural no es necesario si se entiende a todo como 'totalmente'.

Hay por lo menos cuatro ejemplos de cambio de régimen pronominal en que Medina modifica el texto académico; en dos casos $(3,5,4$ predicarles por predicarlos y 7,17,3 baciéndoles por baciéndolos) también contra la tradición textual de las ediciones antiguas consultadas; en 11,42,6 les rompieron por los rompieron se aparta de la edición académica que sigue aquí el texto de $\mathrm{H}$, pero transcribe $\mathrm{A}, \mathrm{B}, \mathrm{C}, \mathrm{F}, \mathrm{G}, \mathrm{I}$; en $15,4,8$ Medina sigue la lectura de G,H,I.

En el aspecto morfológico-sintáctico las divergencias son de diverso tipo; en 25,56,3 esta arte por este arte ofrece la lectura de todas las ediciones anteriores a $\mathrm{H}$, que es la que sigue Ferrer del Río; 32,22,8 Lo que por $L a$ que es concordancia innecesaria pues el verso no es proposición sujeto del verso séptimo de la octava con verbo tácito ser, sino relativa con antecedente vitoria. En 37,30,4 derramen por derraman de $\mathrm{H}$ y Ferrer del Río no es un cambio válido pues no se trata de un verbo principal de la proposición consecutiva iniciada en la octava anterior sino del verbo principal de la proposición de relativo incluida. 
Los cambios léxicos aparecen en construcciones, en sustitución de una o más palabras y en cambios completos de uno o más versos. En su mayor parte las'sustituciones no tienen otro sentido que el de una supuesta mayor claridad, no siempre evidente y en ningún caso parecen justificables.

En 1,34,5 Que el caso es grave es una fusión textual de compromiso que altera el texto de A: Si el caso es grave, y no respeta el de las demás ediciones antiguas y la académica: Seguin el caso; en 10,8,7 (ni gobierno por y gobierno de todas las ediciones antiguas consultadas) y 34,6,5 (No pienses por Ni pienses en H y Ferrer del Río) presentan dos construcciones negativas impecables; en el primer caso se trata de una sustitución para enfatizar la pareja de sinónimos orden y concierto (cf. Keniston, 40.94) y en el segundo caso, opuestamente, no es útil para unir las dos partes de la argumentación de Caupolicán ni para justificar su pedido de clemencia a Reinoso. El cambio en 12,13,3 es otro caso innecesario de sustitución de dentro en por dentro de (Cf. Keniston, 41.32), pero sin. alteración métrica. Nada justifica el cambio de desde poco 'apenas' $(15,63,6)$ de C,G,H,I y Ferrer del Río a desde a poco excepto que es el texto de A y B. En 18,56,2 la supresión de de en armada de gente va contra el texto de todas las ediciones consultadas y se explica porque armada es 'flota' en el texto; es innecesaria la preposición de en sangre de súbito que Medina agrega a 24,49,7 contra la tradición textual pues súbito 'súbitamente' no es infrecuente en autores de la época y hasta hoy; el cambio de régimen para el verbo entrar en $28,36,5$ va contra el texto que aparece en las ediciones antiguas y no se justifica; tampoco es necesaria la preposición de en 9,39,1 Retirados alli se reformaron pues el verso insiste en el tiempo en que los vencidos españoles estuvieron reacondicionándose en Santiago.

Las sustituciones léxicas son poco afortunadas y comprenden cambios de una palabra hasta versos enteros. En algunos casos el sentido del cambio se explica como un esfuerzo para "aclarar" el texto, pero muchas veces el cambio es sencillamente modernización. Esta decisión va contra promesas de equilibrio entre el respeto riguroso al texto original y la necesidad "indispensable"' de cambio; en efecto, w1 un sentido estrictamente filológico, los que fueron llevados a cabo en muy pocos casos parecen corrección de textos erróneos. En cambio, la sustitución de versos enteros permite descubrir las ideas de Medina sobre el texto a seguir, previas a la que finalmente prevaleció cuando el trabajo fue, en diversas etapas, a la imprenta.

Entre los cambios de palabra que también parecen una saludable vuelta a ediciones previas a $\mathrm{H}$ y coinciden con $\mathrm{F}$ notamos $A$ món $(1,10,7)$ en vez del incomprensible $A$ mán de $\mathrm{H}$ o el infortunado Amor de Ferrer del Río; De la hambre en vez del más expresivo De hambres $(15,63,4)$ ya en A, B,C,H; francas leyes $(16,43,3)$, por viejasleyes en $\mathrm{G}, \mathrm{H}, \mathrm{I}$, más sensato que el mudas leyes de. B,C,D; indas regiones $(23,60,3)$ vuelve a la tradición textual corregida en $\mathrm{H}$ (indias) que acata Ferrer del Río; tanto cautamente $(25,34,7)$ por tanto astutamente en las demás ediciones; Los troncos aún $(25,35,4)$ por Las llagas aún de las demás ediciones; a robar comida $(30,47,7)$ por a buscar comida de $\mathrm{G}, \mathrm{H}, \mathrm{I}$ a las que sigue Ferrer del Río.

En otros casos, la sustitución resulta de la preferencia arbitraria de otras ediciones; así, Pero alli más veloz $(5,15,1)$ por Pero por más veloz de $\mathrm{F}, \mathrm{G}, \mathrm{H}, \mathrm{I}$ sólo se explica porque es lectura de B,C; circulo ingenioso $(26,48,6)$ por circulo bermoso de F, G,H,I se funda 
seguramente en que es lectura de B,C y D; A la famosa reina por $A$ la casta fenisa de $\mathrm{E}, \mathrm{F}, \mathrm{H}$, es transcripción de $\mathrm{G}, \mathrm{I}$ que no se justifica, igual que en el caso de Que en la falsa opinión $(32,45,8)$ por Que en la misma opinión.

Hay también un grupo de alteraciones textuales respecto de la edición académica que parecen inexplicables desde el punto de vista filológico pero que sin duda se ajustan a las preferencias personales de Medina. Nada justifica Con osada esperiencia $(5,34,6)$ por el original Con usada excepto que así no aparece el participio del verso siguiente repetido, pero todas las ediciones traen en 5,54,7 osado y la repetición se debe a un cambio originado en la edición académica y, como se ha visto más arriba, totalmente innecesario, pues usado 'acostumbrado' es acepción corriente en la literatura de la época (Garcilaso Egloga I, 79); prevalece $(9,2,2)$ por convalece de todas las ediciones estropea el texto sin justificación evidente; Es este tiempo ... cuando $(9,22,5)$ es la lectura de todas las ediciones consultadas y nada justifica el reemplazo de esta construcción con ser por En este tiempo de Medina (Keniston, 16.41); a que me digas $(32,66,2)$ por a que me sigas no es justificable ni por la tradición textual ni por el sentido de la estrofa.

Quedan, por último, los casos de sustitución parcial o totâl de versos enteros. También en estos casos se puede notar una vuelta a $F$, y hay que interpretar esto como una superposición de la etapa inicial de la empresa editora de Medina sobre el producto final. Según dijimos ya, sin duda fue el propósito inicial editar el texto de F, como se anuncia en el Tomo I, p. VIII, pero el peso del trabajo cuidadoso hecho por Ferrer del Río debió inclinar la decisión final hacia $\mathrm{H}$ que, por otra parte, ofrecía un texto más completo. El pasaje de un texto al otro no impidió que la huella del texto previamente elegido aparecieralinadvertidamente en las decisiones finales. En 3,63,1-6, Medina se aparta de Ferrer del Río y opta por la lectura de $\mathrm{F}$ en donde, por lo demás, se transcribe $\mathrm{A}$; en 25, 14,7-8 Medina transcribe $F$ contra $G, H, I$ a las que sigue Ferrer; en $25,26,7-8$ se reproduce la opción anterior ${ }^{7}$.

Además, hemos notado casos en que Medina se aparta de Ferrer del Río (quien transcribe $\mathrm{F}, \mathrm{H}$ ) y sigue el texto de ediciones anteriores.

Los ejemplos citados son prueba suficiente de que la preparación del texto no solamente pasó por diversas etapas excluyentes sino que el resultado final debe considerarse como la versión Medina de por lo menos tres textos: F,H y la edición de la Academia. Es cierto que Medina advierte al lector (llustración X, "Bibliografía de La Araucana", Tomo IV, p. 52) que " nuestro texto no debe aceptarse, y por lo tanto, el académico, sin las notas lexicográficas que a él dedicamos"; aquí " notas lexicográficas" significa "variantes textuales" pero esta afirmación no alcanza a cumplirse plenamente en la aludida Ilustración de las variantes (Tomo IV, p. 107 y ss.), en parte porque el sistema ideado es extremadamente confuso, en parte porque esta Ilustración ofrece abundantes errores, erratas, traspapelamientos y omisiones, lo que obliga a utilizar tan vasta información con extremo cuidado.

Por otra parte, en el Prólogo a la Ilustración XII (Tomo IV, p. 109) advierte Medina que "las variantes que no tienen al pie indicación de procedencia corresponden a las

${ }^{7}$ También es posible localizar vueltas a F en cambios fonéticos: 18,35,2 (preveniendo) y 25,31,2 (Yanaruna) por ejemplo. 
ediciones príncipes, esto es, la Primera parte, a la de 1569; en la Segunda, a la de 1578; y en la Tercera, finalmente, a la de 1589, todas de Madrid', pero en las variantes a 24,6,2 (p. 388, estrofa 5 de la edición de Medina) se descubre que las variantes de la Segunda parte "que venimos anotando" son las de Zaragoza, 1578 (D en nuestras abreviaturas), por lo que debe deducirse, y es así, que las variantes sin indicación de procedencia hasta el canto 24, correspondientes a la Segunda parte, y en adelante, corresponden no a la princeps sino a D. Además, en el Prólogo al texto del poema, publicado en 1910, se anuncia que "A1 fin del texto consignaremos las variantes que al respecto de la edición que seguimos (sin duda $\mathrm{F}$, entonces) se encuentran en todas las que aparecieron con anterioridad a ella en la Península y que hoy se conocen, a excepción de la primera de la Primera parte que vio la luz pública en Madrid y de que existen solo tres ejemplares en Europa' '(Tomo I, p. VIII). Parece obvio deducir que el Prólogo al texto fue escrito con anterioridad a la preparación y forma final del texto y que un ejemplar de A llegó a manos de Medina, seguramente a través de la reprodución hecha en New York por De Vinne Press en 1902 para la Hispanic Society. También habrá que deducir que la Ilustración X, "Bibliografía de La A raucana", fue escrita después de la Ilustración XIII, porque es en aquélla en donde Medina decide, con buenas razones, que B es la princeps de la'Segunda parte (Tomo IV , p. 9) y que, a pesar de posibles dudas, hay que descartar a D como princeps (Tomo IV, p. 12); no obstante todo esto, y seguramente porque ya no era técnicamente posible un cambio,. $\mathrm{D}$ es considerada la princeps para la llustración XII de las variantes del poema, como ya hemos señalado.

Finalmente, sospechamos que $\mathrm{F}$ dejó de ser leída con cuidadoso escrúpulo una vez hecha la decisión de editar $\mathrm{H}$, a pesar del hecho de que F, para Medina "sin duda fue impresa bajo la inmediata inspección del poeta" (Tomo I, p. VIII) y a pesar de que la consideraba la más autorizada porque fue "la última que él corrigiera antes de morir" (Tomo III, p. 155). Por ejemplo, Medina se equivoca cuando señala que las estrofas 48 a 53 del Canto 32 en E se suprimieron en F (Tomo IV, p. 19); en efecto, las seis citadas estrofas aparecen en esta|primeraledición conjunta de las tres partes, y las irregularidades en las signaturas de las páginas aparecen en $\mathrm{E}$ y en $\mathrm{F}$, por lo que cabe deducir que tal vez se trate de una reimpresión con leves retoques.

En resumen, la edición de Medina, extraordinaria en tantos aspectos, debe manejarse con extrema cautela en lo que al texto se refiere. AquíiMedinalno fue consistente y terminó por crear un texto que refleja criterios filológicos extravagantes si se los compara con el rigor y la erudición con que trató los aspectos históricos y literarios relacionados con el poema. El entusiasta afán por modernizar, revisar, alterar y retocar pudo más que el respetuoso deseo inicial de volver al texto original $(\mathrm{F}$ o H$)$ del poema épico más afortunado de América.

Herbert H. Lehman College, CUNY

ISAIAS LERNER

\footnotetext{
${ }^{8}$ Hemos notado por lo menos 41 cambios entre una y otra edición que, en la mayoría de los casos, tienen que ver con variantes ortográficas.
} 